\title{
Nest Digging by Leaf-Cutting Ants: Effect of Group Size and Functional Structures
}

\author{
Roberto da Silva Camargo, Ricardo Toshio Fujihara, and Luiz Carlos Forti \\ Laboratório de Insetos Sociais-Praga, Departamento de Produção Vegetal, Faculdade de Ciências Agronômicas, UNESP, P.O. Box 237, \\ 18603-970 Botucatu, SP, Brazil \\ Correspondence should be addressed to Roberto da Silva Camargo, camargobotucatu@yahoo.com.br
}

Received 27 April 2011; Revised 25 May 2011; Accepted 6 June 2011

Academic Editor: Fernando Fernández

Copyright ( 12012 Roberto da Silva Camargo et al. This is an open access article distributed under the Creative Commons Attribution License, which permits unrestricted use, distribution, and reproduction in any medium, provided the original work is properly cited.

Leaf-cutting ant workers dig underground chambers, for housing their symbiotic fungus, interconnected by a vast quantity of tunnels whose function is to permit the entrance of food (leaves), gaseous exchanges, and movement of workers, offspring, and the queen. Digging is a task executed by a group of workers, but little is known about the group effect and group-constructed functional structures. Thus, we analyzed the structures formed by worker groups $(5,10,20$, and 40 individuals) of the leaf-cutting ant, Atta sexdens rubropilosa, for 2 days of excavation. The digging arena was the same for the 4 groups, with each group corresponding to a different density. Our results verified a pattern of tunneling by the workers, but no chamber was constructed. The group effect is well known, since the 40-worker group dug significantly more than the groups of 5, 10, and 20. These groups did not differ statistically from each other. Analysis of load/worker verified that workers of the smallest group carried the greatest load. Our paper demonstrates the group effect on the digging of nests, namely, that excavation is proportional to group size, but without emergence of a functional structure such as a chamber.

\section{Introduction}

Medium-sized leaf-cutting ant workers are responsible for digging new functional structures within the nest including chambers and tunnels [1]. These workers respond well to the stimulus to dig even when isolated or in small groups. During digging the workers must be involved in coordinated activity and respond actively to regions excavated by other ants. After the initial construction process is established, nestmates must be recruited to the location of the activity. This recruitment of nest companions is governed by a positive feedback mechanism by means of communication between the initial excavators and the recruits. The signal utilized for the recruitment is probably stridulatorial communication [2], which runs through the substrate (soil) to recruit nestmates to the point of activity. When the density increases, this signal ceases, as does its function.

Concomitant with the growth of the structure, we would find a negative feedback coordinating the activity, in other words, conducting the workers to finalize the task of digging. The workers may perceive this via chemical cues (pheromones), metabolic products of the workers $\left(\mathrm{CO}_{2}\right)$ $[3,4]$, or by the encounter rate among them [5]. The touches among these workers are a product of momentary density in the area of activity, that is, a monitoring of the task [5].

This hypothesis is based on the allocation of tasks without centralized control, in which the individuals respond to simple local cues (signals). This rate of signals depends on group characteristics such as their size and density [5]. Thus, the functional structures emerge from the activity of the ants, ceasing with the enlargement of the required structures, whether they be tunnels or chambers.

It is known that among leaf-cutting ants, when small groups of workers are formed (2 to 8 ) for digging, they do not increase the number of tunnels and instead deepen those already excavated, demonstrating social organization and coordination of work [1]. These indications constitute strong evidence of a nest construction system, although this has not yet been demonstrated experimentally. 


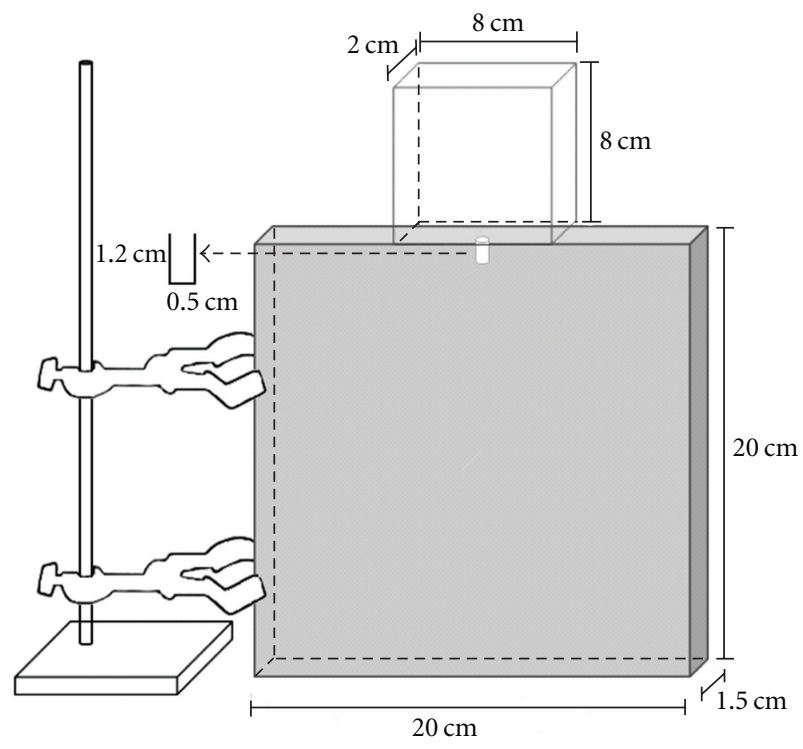

Figure 1: Experimental setup: the workers were allocated into a box with a pregiven tunnel to incite digging. The workers continued to dig for 48 hours.

In the present work we analyzed the structures formed by groups of workers $(5,10,20$ and 40 individuals) of the leafcutting ant, Atta sexdens rubropilosa, for 2 days of digging. The size of the excavation arena was the same for the 4 groups, with each group corresponding to a different density. The excavation arena was disposed vertically, permitting visualization of the digging in a uniform environment. Thus, it was possible to study the group effect on the nest digging by small groups of workers.

\section{Material and Methods}

2.1. Selection of Workers. Twenty-five workers from the field were collected in adult nests during nest digging. These were isolated, labeled, and transported to the laboratory. The following aspects were measured: head width, body mass, and load mass (soil pellet). This enabled patterning of the size of the ants that effectively do the digging in a natural situation.

In the laboratory, workers were selected according to their size class (head width from 1.2 to $1.6 \mathrm{~mm}$ ) in midsized colonies (3 years) maintained in the Laboratory of Insect Pests—FCA/UNESP—Botucatu, São Paulo State, Brazil.

2.2. Excavation Boxes. The fifteen glass boxes utilized had dimensions of $20 \mathrm{~cm}$ in length and height, and $1.5 \mathrm{~cm}$ in thickness. These were filled with soil collected at a depth of $60 \mathrm{~cm}$ and sieved (soil density $=1.6 \mathrm{~g} / \mathrm{cm}^{3}[6]$; water content: $5.4 \%)$. A small tunnel, constructed manually (artificially) in this apparatus, was $1.2 \mathrm{~cm}$ in length and $0.5 \mathrm{~cm}$ in width.

Above this apparatus was placed a small glass box $(8 \times 8$ $\times 2 \mathrm{~cm}$ ) into which was transferred a colony for conducting the experiment (Figure 1).
2.3. Rate of Digging and Structures Formed. We analyzed the structures formed by groups of generalist workers $(5,10$, 20, and 40 individuals) of the leaf-cutting ant, Atta sexdens rubropilosa, for 2 days of digging.

The workers stayed in the digging box for 48 hours, with the excavated soil being removed every 12 hours. The soil volume dug out by the workers in the 12-hour period was collected and dried in an oven for 24 hours at $80^{\circ} \mathrm{C}$.

After the 48-hour digging period, liquid plaster was added to obtain the exact dimensions of the architecture of these constructions. After the plaster had dried, these structures and the soil were removed and carefully analyzed.

The structures were analyzed by measuring the area excavated by the workers on two planes; the structures were photographed and their area calculated in $\mathrm{mm}^{2}$ via the program Image J from the National Institutes of Health, USA., http://rsb.info.nih.gov/ij/. These measures were correlated with the number of workers involved in the excavation, thereby providing a ratio between the number of structures formed and the number of workers.

The volume of these structures was calculated by their weight, with the volume in $\mathrm{g}$, converted to $\mathrm{mL}$.

2.4. Statistical Analyses. A linear regression was applied to correlate the mass of workers with their load in the field, to enable selection of the size class appropriate for digging. The head widths of field workers were compared with those of laboratory workers by the test of Mann-Whitney $(\alpha=0.05)$. The data for the area and volume of excavated soil were submitted to analysis of variance (ANOVA; $\alpha=0.05$ ), with subsequent paired comparison by the methods of StudentNewman-Keuls and Dunn.

\section{Results}

3.1. Selection of Workers. The workers from the field presented a linear relationship between their body mass $(0.004$ $\pm 0.002 \mathrm{~g})$ and the mass of soil pellets $(0.017 \pm 0.01 \mathrm{~g})$ deposited outside the nest ("best-fit" linear equation, $x=$ $0.00125+(0.173 * y), R=0.849,(t=7.702, P<0.001))$ (Figure 2). In their pellets these workers carry on average 4times their body mass (ratio = soil pellet mass/worker mass, mean $=4.05$; standard deviation $=1.66, N=25$ ). These pellets are transported frontally to the exterior of the nest by the mandibles, without projecting all the load mass behind the body, as they do when collecting vegetal matter. This must be the manner by which they fabricate such pellets. The pellets are formed by the behavioral act of "biting" the soil matrix with the mandibles; in other words, the workers remove the soil fragments and aggregate them with the aid of their metathoracic legs. After the soil pellet is formed, it is taken to the exterior of the nest, but is carried frontally, between the mandibles. In unpublished data on leaf-cutting ant queens, we observed that when one of the tibiae of the metathoracic legs is mutilated or cut, these queens become slower and dig more slowly than normal ones. This is due to an inefficiency in aggregating soil to form the soil pellets. 
TABle 1: Digging rate $(\mathrm{g})$, area excavated $\left(\mathrm{mm}^{2}\right)$, and volume of soil excavated $\left(\mathrm{cm}^{3}\right)$ by groups of workers $(5,10,20$, and 40$)$, as a function of elapsed time.

\begin{tabular}{|c|c|c|c|c|c|c|c|c|c|}
\hline \multirow{2}{*}{ Group size } & \multicolumn{5}{|c|}{ Volume of soil (g) } & \multirow{2}{*}{ Load/worker ratio } & \multirow{2}{*}{ Trips } & \multirow{2}{*}{ Area } & \multirow{2}{*}{ Volume } \\
\hline & 12 & 24 & 36 & 48 & total & & & & \\
\hline 5 & 3.0 & 1.5 & 0.0 & 0.0 & 4.5 & 0.90 & 53 & $828.5 \pm 436$ & $7.36 \pm 4.1$ \\
\hline 10 & 4.5 & 1.1 & 0.0 & 0.0 & 5.6 & 0.56 & 33 & $1066.0 \pm 464.64$ & $7.37 \pm 3.5$ \\
\hline 20 & 9.8 & 0.3 & 0.0 & 0.0 & 10.1 & 0.50 & 30 & $1176.5 \pm 461.01$ & $11.40 \pm 4.5$ \\
\hline 40 & 21.6 & 1.7 & 1.1 & 2.4 & 26.9 & 0.67 & 40 & $4530.4 \pm 1863.67$ & $36.33 \pm 12.7$ \\
\hline
\end{tabular}

${ }^{*}$ Load/worker ratio = total soil volume/number of workers in the group; ${ }^{*}$ trips $=($ load/worker ratio $) / 0.017$ (average mass of soil pellets).

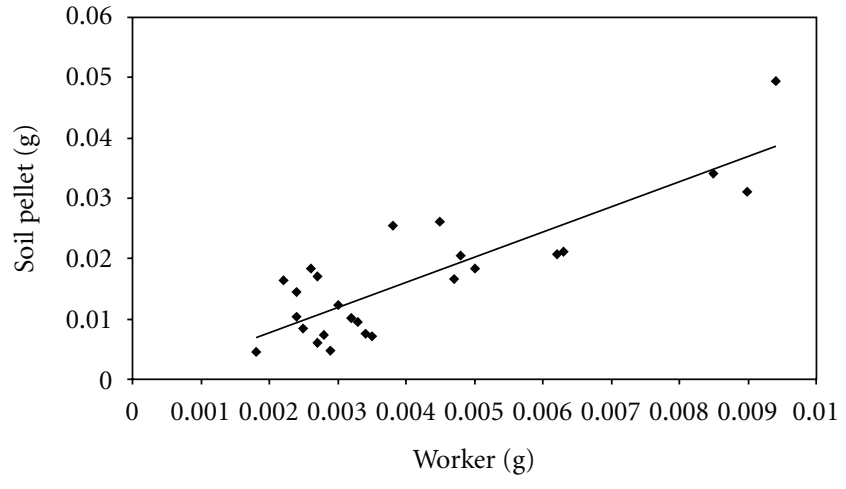

FIGURE 2: Ratio between mass of workers and their soil load in a natural situation. Linear regression was applied with the "best-fit" linear equation $(x=0.00125+(0.173 * y), R=0.849,(t=7.702$, d.f. $=24, P<0.001)$ ).

In addition to mass, the head width was measured in both field and laboratory workers. The field workers presented a head width of $1.5 \pm 0.3 \mathrm{~mm}$ while those of the laboratory did not differ significantly at $1.4 \pm 0.5 \mathrm{~mm}(U=568.500$, $P=0.174$ ). Thus, it may be stated that workers from the field and laboratory were similar, belonging to the same class size, which we can denominate the generalists ([7], on head width from 1.2 to $1.6 \mathrm{~mm}$ ).

3.2. Digging Rate and Structures Formed. The highest digging rate occurred in the first 12 hours after the release of the workers, and decreased over time (Table 1). There was a significant difference among the times (Kruskal-Wallis test, $H=10.727$, d.f. $=3, P<0.05$ ) while the posttest (Student-Newman-Keuls) determined that the volume of soil excavated in 12 hours differed significantly from the other evaluation periods.

We calculated that each worker transported from 0.50 to $0.90 \mathrm{~g}$ of soil throughout the experiment, within each group. The trips were calculated according to the average volume of soil that each worker transported per trip $(0.017 \mathrm{~g})$, resulting in 30 to 53 trips during the experiment (Table 1).

In relation to the structures formed by groups, we verified a specific tunneling pattern by the workers (Figure 3). The area excavated by the groups presented a significant difference (Kruskal-Wallis test, $H=34.697$, d.f. $=3, P<$ 0.001) while the posttest (Student-Newman-Keuls) determined that the 40-worker group dug significantly more than the groups of 5, 10, and 20. These groups did not differ significantly from each other.

The volume of structures created by the workers, determined by the plaster molds, differed significantly among the groups (Kruskal-Wallis test, $H=36.359$, d.f. $=3$, $P<0.001$ ), while the posttest (Dunn) showed that the 40worker group dug significantly more than the 5-, 10-, and 20 -worker groups. There was no significant difference among these groups.

\section{Discussion}

The results obtained by the present study verified that the workers dug tunnels, but no chamber to house them (Figure 3). The emergence of a functional structure, such as a chamber, is only possible in leaf-cutting ants when the worker group is larger and symbiotic fungus is present as a stimulus for its construction. Fröhle and Roces [8] verified the emergence of functional structures, including chambers, in larger groups (750, 1500, 2500 workers), with diverse volumes of fungus. These workers were apt to enlarge a fungus chamber to house a greater fungus volume, independent of the number of ants.

A higher digging rate was observed at the beginning of the experiment, followed by a reduction as time elapsed (Table 1), as already observed in other species $[4,8,9]$. According to Buhl et al. [9], this decrease in activity at the end of the digging dynamic can be explained by two possible mechanisms: the first corresponds to a reduction in activity by means of a perception of specific signals from individuals such as the concentration of $\mathrm{CO}_{2}$ [10] or the rate of encounters among ants [11]. The second relies on the propagation properties of the recruitment, leading to a stopping activity according to the determinate density of ants [12].

The volume of excavated soil varies directly with the number of ants in the group (Table 1); however, almost all of the ants were observed to dig. Nevertheless, it is known that in small groups the workers dig more than those in larger groups, as explained by Fröhle and Roces [8]. This is due to the population density; in other words, the smaller the group, the more possibilities the workers will have to dig and all are recruited with opportunities to execute their task.

In summary, our study demonstrates the group effect on the digging of nests, namely, that digging is proportional to group size, but without emergence of a functional structure such as a chamber. 


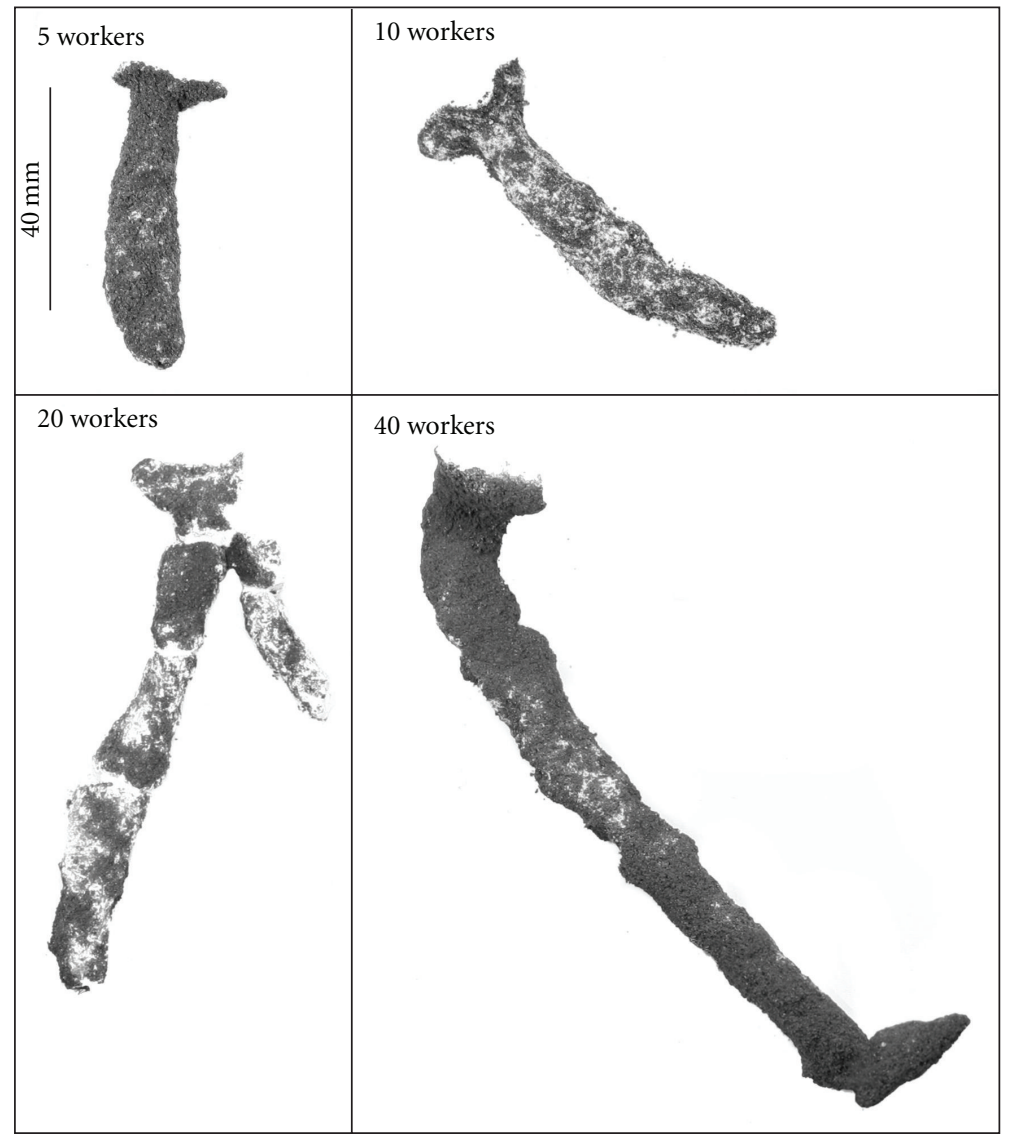

FIGURE 3: Structures produced by groups of Atta sexdens rubropilosa workers, in groups of 5, 10, 20, and 40, throughout 48 hours.

\section{Acknowledgment}

The authors would like to thank "Fundação de Amparo à Pesquisa do Estado de São Paulo" (FAPESP) for the postdoctoral scholarship to the first author (2007/04010-0) and for a research aid (2007/07091-0).

\section{References}

[1] M. M. P. Rodrigues and F. L. Ribeiro, "Organização da escavação em obreiras de Atta sexdens rubropilosa: Atração por túneis iniciados," in I Encontro Paulista de Etologia, p. 242, São Paulo, Brazil, 1983.

[2] H. Markl, "Stridulation in leaf-cutting ants," Science, vol. 149, no. 3690, pp. 1392-1393, 1965.

[3] P. P. Hangartner, "Carbon dioxide, a releaser for digging behavior in Solenopsis geminata (Hymenoptera: Formicidae)," Psyche, vol. 76, no. 1, pp. 58-67, 1969.

[4] P. Rasse and J. L. Deneubourg, "Dynamics of nest excavation and nest size regulation of Lasius niger (Hymenoptera: Formicidae)," Journal of Insect Behavior, vol. 14, no. 4, pp. 433449, 2001.

[5] D. M. Gordon and N. J. Mehdiabadi, "Encounter rate and task allocation in harvester ants," Behavioral Ecology and Sociobiology, vol. 45, no. 5, pp. 370-377, 1999.

[6] C. Stein and G. F. Xavier, "Does substrate density affect the nest depth of female Atta sexdens rubropilosa (Forel, 1908)
(Hymenoptera, formicidae)," Brazilian Journal of Medical and Biological Research, vol. 17, pp. 297-300, 1984.

[7] E. O. Wilson, "Caste and division of labor in leaf-cutter ants (Hymenoptera: Formicidae: Atta)-I: the overall pattern in A. sexdens," Behavioral Ecology and Sociobiology, vol. 7, no. 2, pp. 143-156, 1980.

[8] K. Fröhle and F. Roces, "Underground agriculture: the control of nest size in fungus-growing ants," in From Insect Nest to Human Architecture, G. Theraulaz, R. Solé, and P. Kuntz, Eds., pp. 95-104, Venice, Italy, 2009.

[9] J. Buhl, J. Gautrais, J. L. Deneubourg, and G. Theraulaz, "Nest excavation in ants: group size effects on the size and structure of tunneling networks," Naturwissenschaften, vol. 91, no. 12, pp. 602-606, 2004.

[10] M. D. Cox and G. B. Blanchard, "Gaseous templates in ant nests," Journal of Theoretical Biology, vol. 204, no. 2, pp. 223238, 2000.

[11] D. M. Gordon, R. E. Paul, and K. Thorpe, "What is the function of encounter patterns in ant colonies?" Animal Behaviour, vol. 45, no. 6, pp. 1083-1100, 1993.

[12] J. L. Deneubourg and N. R. Franks, "Collective control without explicit coding: the case of communal nest excavation," Journal of Insect Behavior, vol. 8, no. 4, pp. 417-432, 1995. 

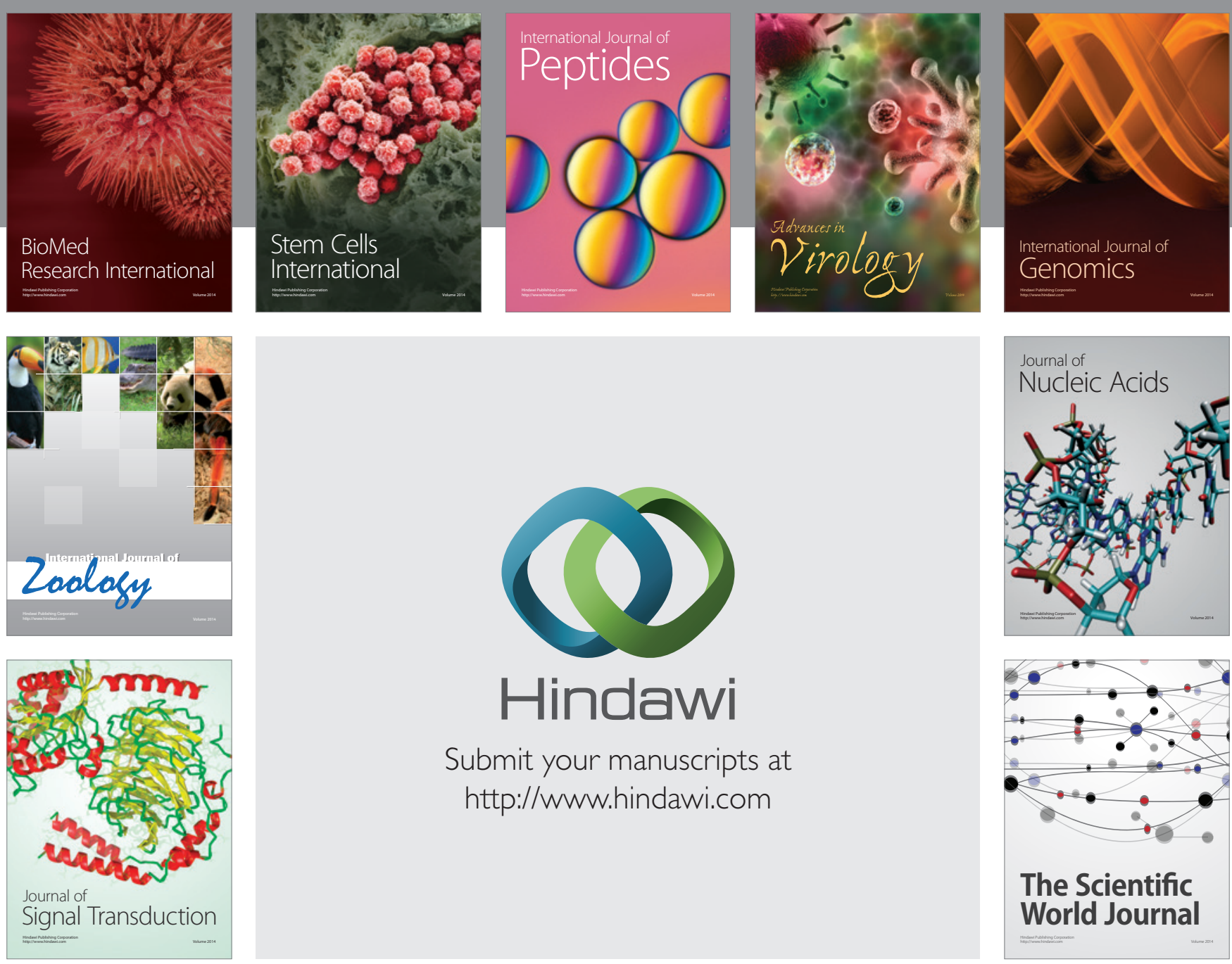

Submit your manuscripts at

http://www.hindawi.com
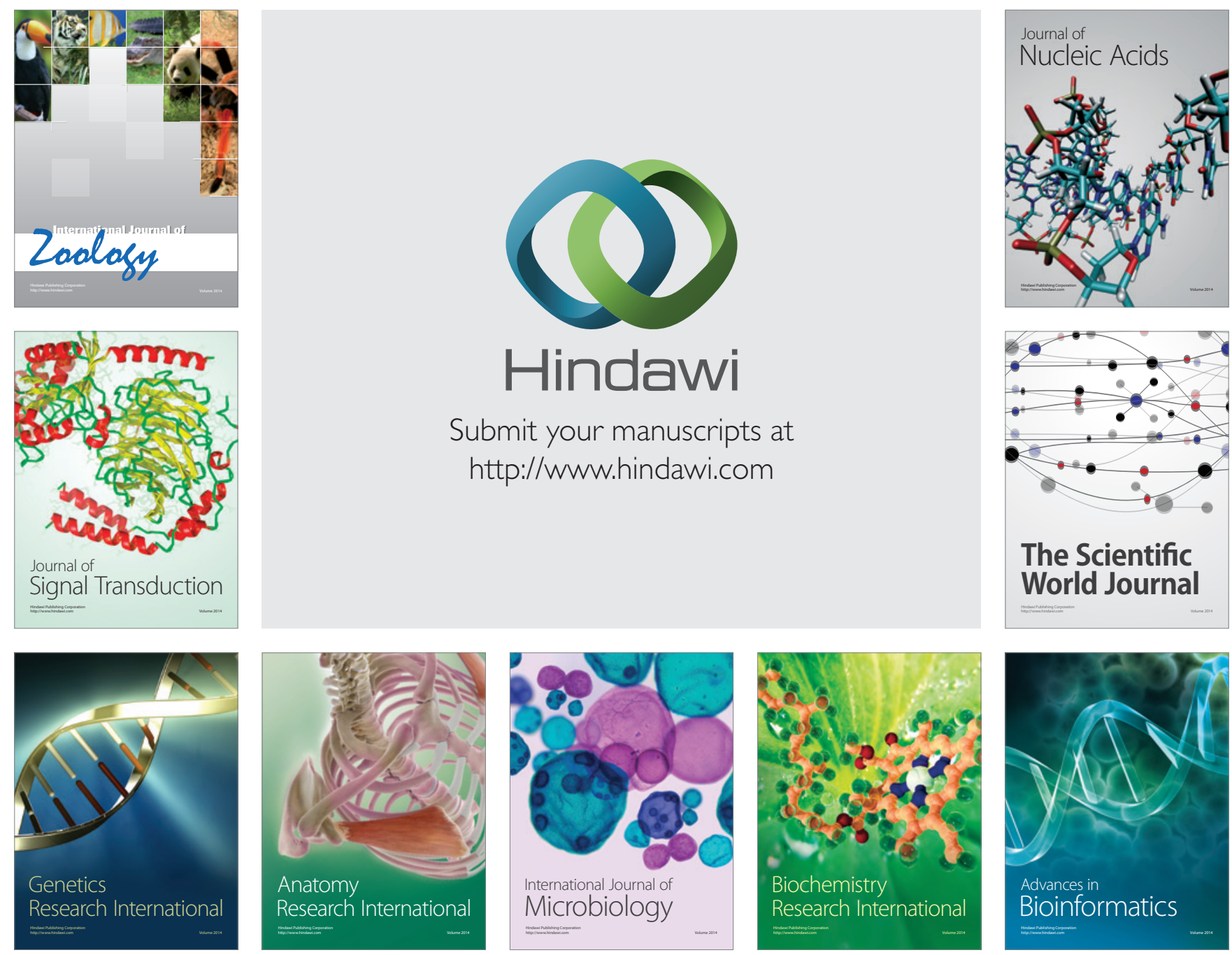

The Scientific World Journal
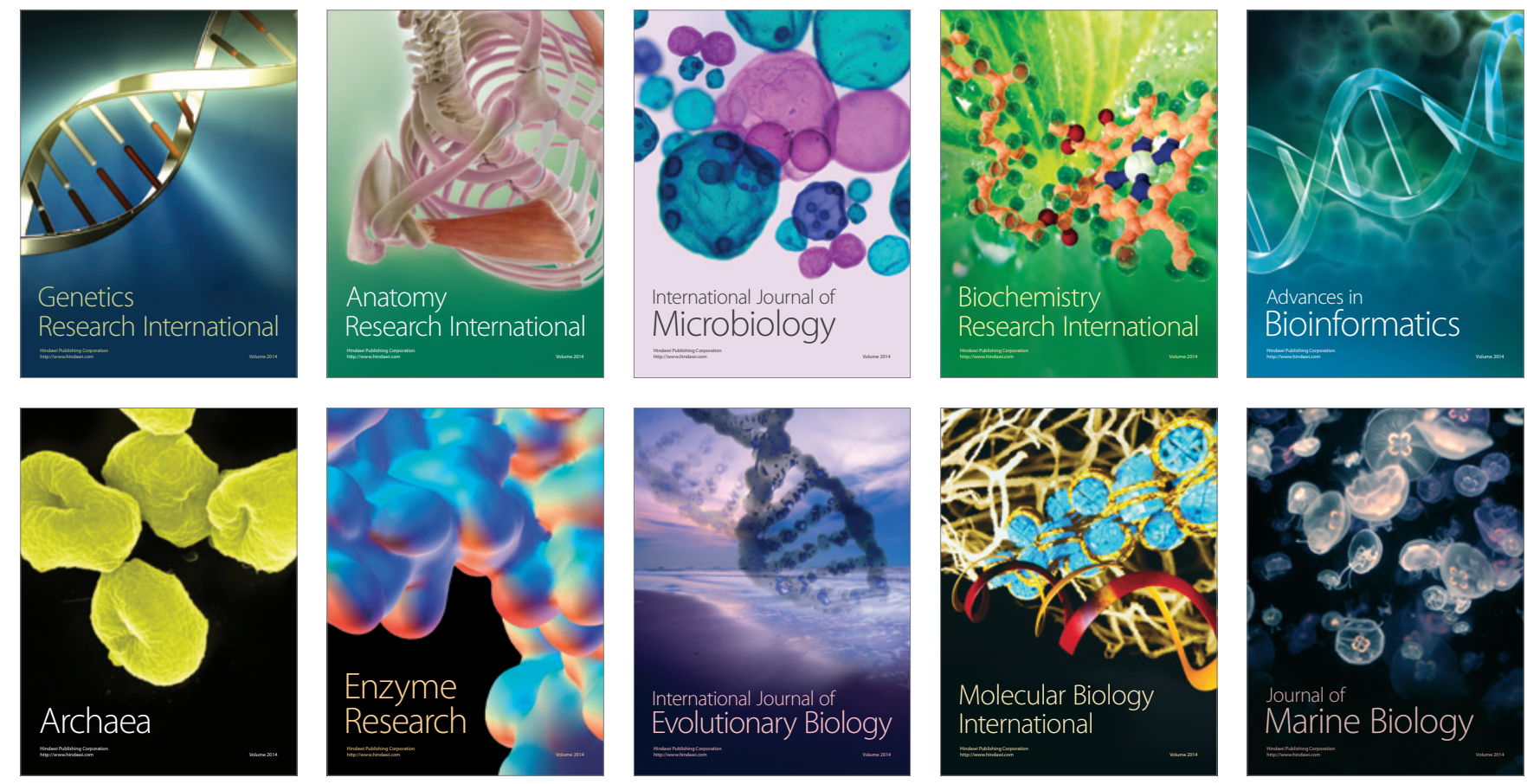